$>$ Combinant une connaissance grandissante des processus de neurogenèse et de spécification neuronale aux découvertes récentes des mécanismes moléculaires et épigénétiques qui gouvernent l'acquisition et le maintien de l'identité cellulaire, les approches de programmation de cellules pluripotentes et de reprogrammation de cellules somatiques en neurones à des fins thérapeutiques se multiplient. Dans cette synthèse, nous revenons sur les travaux qui ont permis l'obtention de neurones à partir de cellules cérébrales en culture, et discutons les très récents résultats de conversion directe de cellules neurales in vivo, qui constituent désormais les fondements de la régénération induite du système nerveux central par ses propres cellules. <

Caractérisé par une impressionnante diversité cellulaire et l'extrême complexité de ses réseaux neuronaux, le système nerveux central (SNC) se définit également par son incapacité quasi totale à se régénérer. Les sources de dommages sont pourtant nombreuses: vieillissement, traumatismes, accidents vasculaires, pathologies neurodégénératives et démyélinisantes. À l'issue du développement, la neurogenèse foisonnante des premiers temps subit de telles contraintes (quantitatives, qualitatives, anatomiques) qu'on l'a longtemps crue complètement éteinte chez l'adulte. Deux régions restreintes du cerveau adulte continuent néanmoins de produire une quantité limitée de neurones: la zone sous-ventriculaire, qui borde le ventricule latéral et génère les interneurones des bulbes olfactifs, et la zone sous-granulaire du gyrus denté, qui génère les neurones granulaires hippocampiques [1]. La mobilisation de ces cellules souches neurales à des fins régénératives reste cependant extrêmement limitée [2]. Dans ces conditions, de nouvelles stratégies thérapeutiques visant à remplacer les neurones perdus ont récemment vu le jour. Combinant une connaissance grandissante des processus de neuro-

\section{Vers une régénération induite du système nerveux}

\section{Reprogrammation des cellules somatiques endogènes en neurones}

Christophe Heinrich ${ }^{1}$, Caroline Rouaux ${ }^{2}$

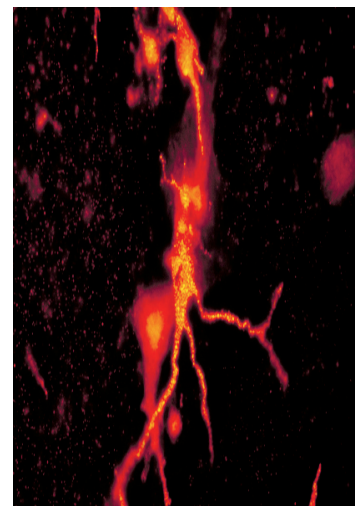

${ }^{1}$ Inserm U836, institut des neurosciences de Grenoble, centre de recherche Inserm U836-UJF-CHU, université Joseph Fourier, 38042 Grenoble Cedex 9 , France ;

${ }^{2}$ Inserm U1118, fédération de médecine translationnelle de Strasbourg, université de Strasbourg, 67085 Strasbourg, France.

christophe.heinrich@ujf-grenoble.fr caroline.rouaux@inserm.fr

genèse et de spécification neuronale aux découvertes plus récentes des mécanismes moléculaires et épigénétiques gouvernant l'identité cellulaire, les approches de programmation de cellules pluripotentes (cellules souches embryonnaires ou cellules pluripotentes induites) et de reprogrammation de cellules somatiques sont à présent en plein essor. L'évolution rapide de cet axe de recherche émergent permet d'ores et déjà d'induire la génération de neurones, in vivo, par reprogrammation de cellules cérébrales endogènes. Ici, nous proposons une synthèse des études les plus récentes qui permettent aujourd'hui d'envisager une thérapie cellulaire basée sur la génération, au sein même du SNC, de populations neuronales d'intérêt thérapeutique à partir de cellules somatiques endogènes : vers une « régénération induite » du SNC.

\section{Différenciation, reprogrammation et conversion directe}

De la cellule souche au neurone, du fibroblaste à la cellule souche La génération de neurones à des fins thérapeutiques a vu le jour au cours des deux dernières décennies du $x x^{e}$ siècle. Ces premiers travaux ont notamment permis de tester divers modes (suspension, monocouche, gouttes, etc.) et milieux de culture, pour induire la génération de cellules au phénotype neuronal, à partir de cellules souches. C'est au tout début des années 2000 que la première différenciation in vitro de motoneurones spinaux (MNS) à partir de cellules souches embryonnaires a été rapportée [3]. Hynek Wichterle et Tom Jessell se sont servis des mêmes signaux extracellulaires qui, au cours du 
développement, instruisent la spécification des MNS à partir des progéniteurs de la moelle épinière [3]. Rapidement, les facteurs de transcription ont pris le relais des signaux extrinsèques pour programmer les cellules souches embryonnaires en divers types neuronaux [4, 5]. Dans le même temps, Kazutoshi Takahashi et Shinya Yamanaka découvraient la reprogrammation nucléaire, démontrant que l'expression forcée de combinaisons de facteurs de transcription suffisait à reprogrammer une cellule somatique, en l'occurrence un fibroblaste, en une cellule pluripotente induite (iPSC, induced pluripotent stem cell) [6]. Couronnés par le prix Nobel de médecine, ces travaux ont contribué à faire germer l'idée qu'une cellule pleinement différenciée garde la possibilité de changer d'identité, et potentiellement d'acquérir, en réponse à des facteurs intrinsèques forts, les caractéristiques d'un autre type cellulaire, sans nécessairement repasser par un stade de pluripotence. Le concept de conversion directe/reprogrammation dirigée était né. Historiquement pourtant, l'idée n'était pas neuve. $\varepsilon n$ effet, l'équipe d'Harold Weintraub avait, dès la fin des années 1980, rapporté la conversion directe de divers types de cellules somatiques (fibroblastes et autres types cellulaires) en myoblastes et myocytes contractiles in vitro, en réponse au facteur de transcription MyoD [7-9]. Dans la même veine, mais plus récemment, les travaux de Thomas Graf ont démontré le rôle des facteurs de transcription C/EBP (CCAAT/enhancer binding protein) alpha et bêta et PU.l (purine-rich DNA sequence) dans la conversion de lymphocytes $B$ et fibroblastes en macrophages $[10,11]$.

\section{Du fibroblaste au neurone}

Dans leur ensemble, ces travaux pionniers ont permis d'envisager la génération de neurones à partir de cellules somatiques, par conversion/reprogrammation directe. C'est en 2010 que l'équipe de Marius Wernig a rapporté l'obtention des premiers «neurones induits » à partir de fibroblastes. En testant méthodiquement divers facteurs de transcription connus pour leur rôle neurogénique, l'équipe a identifié trois gènes, Ascll (Achaete-scute homolog 1), Brn2 (Pou3f2, POU domain, class 3, transcription factor 2) et Mytll (myelin transcription factor 1 -like), dont l'expression combinée suffit à reprogrammer directement les fibroblastes en neurones [12]. Depuis cette étude pionnière, les approches de conversion directe de cellules somatiques en neurones, en réponse à divers cocktails de facteurs de transcription (et plus récemment de microARN) se sont rapidement multipliées, à partir de cellules murines et humaines [13]. À I'heure actuelle, si la génération de divers sous-types neuronaux, et non plus d'un phénotype pan-neuronal, est rapportée de plus en plus souvent, il reste encore à définir les critères précis qui permettront de compléter l'analyse de l'identité de ces neurones induits : à l'interface des marqueurs moléculaires, des profils électrophysiologiques et des connexions axonales [14].

Ces tout premiers travaux de programmation et reprogrammation orientées vers la génération de neurones in vitro ont démontré le rôle incomparable des facteurs de transcription dans l'établissement d'une identité cellulaire donnée, et permis d'identifier les meilleurs candidats à l'induction d'un phénotype neuronal à partir de cellules non neurales. Pour l'heure, la génération de neurones à partir de cellules pluripotentes induites, basée majoritairement, mais pas uniquement, sur l'utilisation de facteurs extrinsèques, reste plus efficace que la reprogrammation directe de fibroblastes en neurones, et permet d'obtenir une plus large gamme de sous-populations neuronales, notamment des neurones corticaux $[15,16]$. Le domaine plus récent de la génération directe de neurones à partir de fibroblastes, basée sur l'utilisation de facteurs intrinsèques (facteurs de transcription en majorité, mais aussi microARN), a néanmoins permis l'obtention de sous-types neuronaux d'intérêt thérapeutique à partir de cellules de patients [17-19]. L'identification d'un plus grand nombre de ces facteurs de transcription clés (ou de combinaisons de ces facteurs) d'une part, et des mécanismes moléculaires, notamment épigénétiques, qui sous-tendent ou à l'inverse bloquent le processus de reprogrammation directe d'autre part [20], permettront à terme d'élargir le panel de sous-types neuronaux dérivés de fibroblastes. Ces méthodes, directes ou indirectes, ont d'ores et déjà permis l'obtention de populations neuronales d'intérêt thérapeutique, et offrent la possibilité d'étudier, de manière simplifiée in vitro, les mécanismes moléculaires responsables de leur vulnérabilité, spécifique au processus de neurodégénérescence.

Cependant, l'application de telles approches à des fins régénératives semble encore difficile à mettre en place. La transplantation de neurones obtenus in vitro n'est en effet pas synonyme de survie, d'intégration et de restauration des fonctions neuronales perdues, surtout lorsqu'elle est envisagée dans le contexte extracellulaire du cerveau adulte pathologique. Si cette stratégie s'est avérée bénéfique dans un modèle murin de maladie de Parkinson [21, 22], I'approche chirurgicale apparaît plus délicate à mettre en œuvre chez l'homme. Dans le cas de la maladie de Parkinson, la transplantation de tissu mésencéphalique ventral (enrichi en progéniteurs dopaminergiques), provenant de fœtus humains, a produit des résultats mitigés mais néanmoins prometteurs [23]. L'un des enjeux majeurs d'une telle approche réside donc, dans un premier temps, dans la survie de cellules pleinement différenciées puis transplantées (par exemple des neurones dopaminergiques obtenus in vitro), là où des progéniteurs neuronaux (par exemple des progéniteurs dopaminergiques fœtaux) peinent à survivre à long terme, en dépit des traitements immunosuppresseurs utilisés pour prévenir les rejets d'allogreffes. Une manière de contourner les difficultés inhérentes à 


\begin{tabular}{|c|c|c|c|}
\hline Cellule d'origine & Facteurs de transcription & Phénotype des neurones induits & Références \\
\hline \multirow[t]{6}{*}{ Astrocyte murin } & Pax6 & Sans phénotype caractérisé & {$[26,27]$} \\
\hline & Neurog2 & Glutamatergique & {$[28,29,31]$} \\
\hline & Dlx2 & GABAergique & {$[28,29]$} \\
\hline & Dlx2, Ascll & GABAergique & {$[28,29]$} \\
\hline & Ascll, Lmxlb, Nurrl & Dopaminergique & [32] \\
\hline & NeuroDl & Glutamatergique & [33] \\
\hline Astrocyte humain & NeuroDl & Glutamatergique & [33] \\
\hline \multirow[t]{2}{*}{ Cellules gliales NG2 murines } & Neurog2 & Glutamatergique & {$[31]$} \\
\hline & NeuroDl & Glutamatergique et GABAergique & [33] \\
\hline Péricyte humain & Ascll, Sox2 & GABAergique & [34] \\
\hline
\end{tabular}

Tableau I. Conversion directe de cellules gliales et péricytes en neurones in vitro.

la transplantation de neurones induits in vitro est d'identifier les cellules qui, au sein même du SNC adulte, pourraient servir de point de départ à la génération de nouveaux neurones.

\section{Les glies, candidats favoris à la reprogrammation neuronale}

Parmi les candidats potentiels à une reprogrammation in situ en neurones, les cellules gliales arrivent en tête de liste. Les glies (astrocytes, oligodendrocytes et microglies) représentent près de $90 \%$ des cellules du SNC, et en peuplent toutes les structures. En outre, elles sont les seules cellules neurales différenciées adultes encore capables de se diviser, même si leurs mitoses restent peu fréquentes dans le SNC sain. À l'inverse, la plupart des conditions pathologiques affectant le SNC, accidents vasculaires cérébraux, traumatismes, gliomes et pathologies neurodégénératives, s'accompagnent d'une gliose réactionnelle, une activation des glies qui se traduit par une prolifération et une hypertrophie $[24,25]$. Si la gliose réactionnelle constitue un système de défense contre les microorganismes et les toxines libérées par le tissu endommagé, elle ne peut pour autant se résumer à ses aspects bénéfiques. La «cicatrice gliale » qui en résulte constitue en effet un frein à la repousse axonale et au rétablissement des fonctions nerveuses lésées. Reprogrammer les glies réactionnelles apparaît donc doublement prometteur : limiter la cicatrice gliale tout en régénérant les neurones perdus.

Pionnières depuis plus de dix ans dans le domaine de la reprogrammation de cellules gliales en neurones, les équipes de Magdalena Götz et de Benedikt Berninger ont initialement démontré la faisabilité d'une telle approche in vitro (Tableau l). Leur première étude rapportait la génération de neurones immatures à partir d'astrocytes isolés du cortex de jeunes souris en réponse à l'expression d'un seul facteur de transcription, Pax6 [26]. Les facteurs de transcription neurogéniques Neurog2 (Neurogenin-2) et Ascll se sont par la suite avérés plus efficaces que Pax6, permettant l'obtention de neurones capables de générer des potentiels d'action [27]. Depuis, nos travaux ont montré que l'utilisation sélective de $\mathrm{Neu}$ rog2 d'une part et d'Ascll et/ou Dlx2 (distal-less homeobox2) d'autre part, permet d'obtenir respectivement des neurones glutamatergiques ou GABAergiques fonctionnels, présentant toutes les caractéristiques électrophysiologiques de neurones adultes, capables en particulier de recevoir et d'émettre des connexions synaptiques [28-30]. Ces résultats ont été reproduits à partir d'astrocytes de jeunes rats en réponse à Neurog2 [31]. Par ailleurs, nous avons observé que l'efficacité de la reprogrammation astrocyte-neurone peut encore être augmentée par l'addition du facteur de transcription Sox2 [28]. À l'instar des études menées sur les fibroblastes, d'autres groupes ont rapporté l'obtention sélective de populations de neurones dopaminergiques ou glutamatergiques à partir d'astrocytes primaires de souris ou d'une lignée d'astrocytes humains, en réponse à différentes combinaisons de facteurs de transcription $[32,33]$ (Tableau I). En parallèle de ces travaux ciblant les astrocytes, d'autres ont décrit l'obtention de neurones glutamatergiques et GABAergiques par conversion directe de cellules gliales NG2 (NG2 glia, progéniteurs d'oligodendrocytes immunoréactifs pour NG2) de souris et de rats $[31,33]$ (Tableau 1), faisant des divers sous-types de glies autant de candidats potentiels à la génération de néo-neurones in vivo.

Afin de valider notre approche chez l'homme, nous avons tenté de reproduire la conversion astrocytesneurones sur des cellules de cortex humain cultivées à partir de prélèvements chirurgicaux [34]. De manière inattendue, les cellules cultivées se sont avérées être des péricytes, une population cellulaire non gliale, 
localisée au niveau des capillaires sanguins et impliquée dans le contrôle du débit sanguin, de l'homéostasie de la barrière hématoencéphalique et de la formation du tissu cicatriciel en réponse à une lésion traumatique [35]. Nous avons ainsi démontré que les péricytes humains peuvent également donner naissance à des neurones en réponse à Ascll et Sox2 [34] (Tableau I).

Astrocytes, cellules gliales NG2 et péricytes, tous présents dans le cerveau adulte, présentent donc la capacité, en culture, de donner naissance à des neurones induits lorsqu'ils sont correctement manipulés. Ces travaux ont permis en outre d'identifier les facteurs de transcription susceptibles d'induire la conversion de cellules somatiques en neurones fonctionnels in vivo.

\section{Régénération induite du SNC ou reprogrammation directe glie-neurone in situ}

La génération de neurones d'intérêt thérapeutique par conversion directe in situ, au sein du SNC en est encore à ses débuts. Cependant, les tout premiers travaux publiés au cours de l'année 2013 apportent la preuve de concept qui valide cette approche, non seulement dans le cerveau sain, mais également dans des modèles de cerveaux lésés. L'équipe de Malin Parmar a démontré que des astrocytes quiescents du striatum pouvaient adopter, in vivo, une identité neuronale en réponse à Ascll, Brn2 et Mytll [36], la combinaison de facteurs de transcription initialement décrite par l'équipe de Wernig dans le processus de conversion fibroblasteneurone [12]. Parallèlement, Chun-Li Zhang et son groupe ciblaient les mêmes cellules avec Sox2 [37], un facteur de transcription neurogénique dont l'expression est maintenue dans les cellules souches neurales [38]. Les auteurs rapportent que Sox2 permet, à lui seul, d'induire la reprogrammation in vivo d'astrocytes quiescents en neuroblastes (exprimant le gène codant pour la doublecortine, une protéine impliquée dans la migration des jeunes neurones postmitotiques), mais que le maintien de son expression empêche leur maturation en neurones pleinement différenciés. Celle-ci requiert encore deux étapes : la répression subséquente de Sox2, et l'ajout de facteurs neurotrophiques tels que le BDNF (brain derived neurotrophic factor), ou le valproate de sodium (VPA, originellement décrit comme inhibiteur de la GABA transaminase et prescrit comme anti-épileptique, reconnu depuis également pour son action épigénétique comme inhibiteur d'histone déacétylases). Les auteurs ont ainsi pu obtenir, in situ, des neurones fonctionnels capables non seulement de générer des potentiels d'action, mais également de recevoir des connexions synaptiques de leurs homologues endogènes [37]. Cette étude démontre la capacité des neurones dérivés d'astrocytes à intégrer un réseau déjà formé, condition sine qua non de leur survie à long terme et de leur potentiel à assurer les fonctions relatives à leur identité nouvellement acquise. Si l'étude de Zhang conduite sur les astrocytes du striatum a permis l'obtention de neuroblastes en réponse à Sox2, nos propres travaux, réalisés dans le cortex cérébral avec ce même facteur de transcription, n'ont pas permis la conversion d'astrocytes ou de cellules gliales NG2
[39] (Figure 1A). Cela suggère un rôle non négligeable du microenvironnement, et/ou révèle l'existence de sous-types astrocytaires spécifiques des différentes structures cérébrales, plus ou moins à même d'être reprogrammés.

Bien que les études de Parmar et de Zhang rapportent avoir ciblé des astrocytes quiescents, il est intéressant de noter qu'elles sont toutes deux basées sur une approche chirurgicale, et l'emploi inévitable d'une canule pour injecter les virus encodant les facteurs de transcription choisis. En d'autres termes, les auteurs ont réalisé une lésion pour délivrer ces agents, et il est possible que cette lésion, si minime soit-elle, ait induit l'activation de cellules gliales avoisinantes. De fait, les néo-neurones ont en majorité été retrouvés le long de la ligne de passage de la canule [36, 37]. Il est donc tout à fait envisageable que les cellules reprogrammées aient eu pour origine des astrocytes réactionnels plutôt que quiescents. C'est en utilisant une approche chirurgicale identique que l'équipe de Mataso Nakafuku rapporte l'obtention de néo-neurones dans un cerveau cette fois décrit comme lésé, en réponse au facteur de transcription Neurog2 et à l'addition des facteurs de croissance FGF2 (fibrobalst growth factor) et EGF (epithelial growth factor) dans le cortex et le striatum [40]. Si les auteurs n'ont pas identifié l'origine cellulaire des candidats à la reprogrammation, l'hypothèse des glies réactionnelles vient immédiatement à l'esprit. De manière intéressante, ils observent que la reprogrammation est plus efficace dans le striatum que dans le cortex, et que l'identité des neurones générés varie en fonction de la structure ciblée: GABAergique dans le striatum, glutamatergique dans le cortex [40] (Figure 1A). Ceci révèle à nouveau l'influence de l'environnement sur la production et la spécification des néo-neurones, et suggère que de nouvelles stratégies devront être mises en place pour garantir l'efficacité des facteurs intrinsèques et minimiser la variabilité due aux facteurs extrinsèques. L'étude plus complète du groupe de Gong Chen démontre la conversion directe d'astrocytes et de cellules gliales NG2 corticales en neurones, en réponse à un seul facteur de transcription, NeuroDl (neurogenic differentiation 1), dans un modèle de lésion induite par l'utilisation d'une canule pour délivrer un virus encodant NeuroD1 [33]. Ces néo-neurones expriment en outre les marqueurs corticaux Tbrl (T-box, brain, 1) et Ctip2 (Bclllb, B cell leukemia/lymphoma $11 B$ ), et sont capables de générer des potentiels d'action et de recevoir des connexions synaptiques de neurones endogènes voisins [33]. 


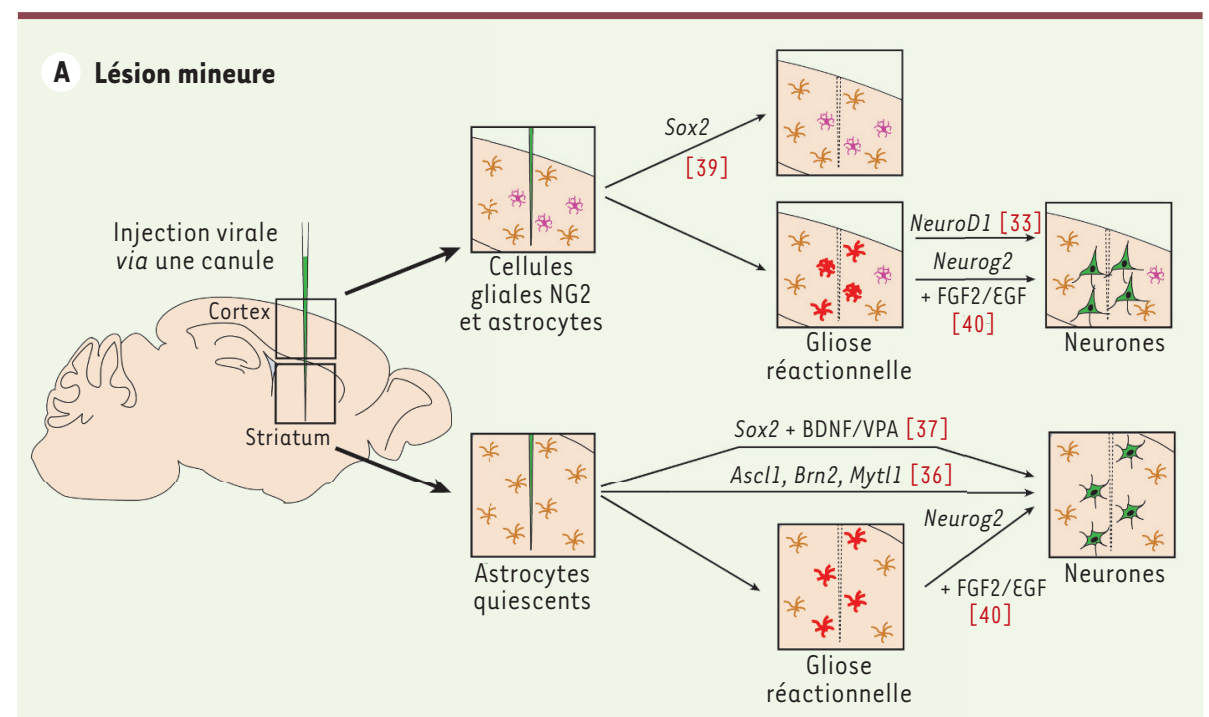

B Gliose réactionnelle massive
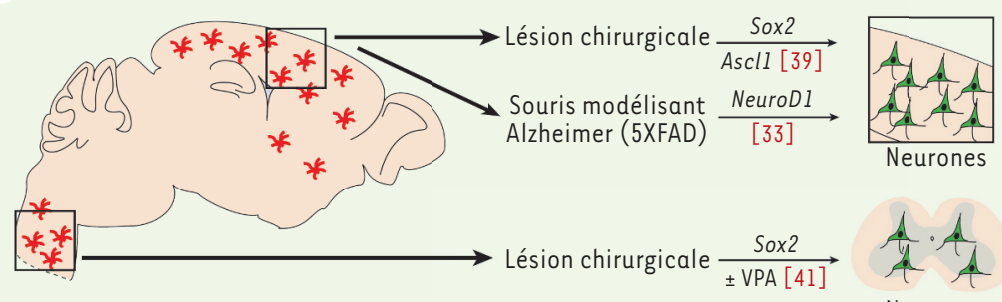

Neurones

\section{Conversion directe de neurones}

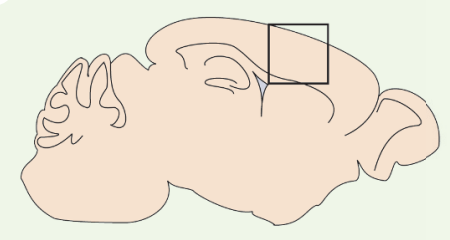

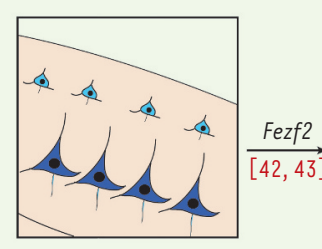

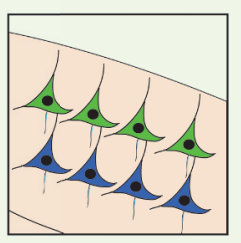

Figure 1. Premiers exemples de conversion directe de cellules neurales en neurones in vivo.

A. Reprogrammation d'astrocytes et de cellules gliales NG2 dans le striatum et le cortex cérébral en conditions de lésion légère, uniquement induite par la canule utilisée pour injecter les virus encodant les facteurs de transcription. $\boldsymbol{B}$. Génération de neurones en conditions de gliose réactionnelle massive induite par une lésion chirurgicale ou dans un contexte neurodégénératif. $C$. Conversion directe de neurones calleux postmitotiques des couches supérieures du cortex cérébral, ou de neurones stellaires de la couche IV, en neurones corticofugaux des couches corticales profondes en réponse à la surexpression du facteur de transcription Fezf2, in vivo.

\section{Reprogrammation directe des glies en conditions pathologiques in vivo}

Faisant un pas de plus vers l'approche régénérative, Guo et al. ont testé la reprogrammation directe d'astrocytes par NeuroDl dans le cortex de souris 5XFAD, un modèle de maladie d'Alzheimer notamment caractérisé par une gliose réactionnelle massive [33]. De manière remarquable, ils démontrent non seulement que la reprogrammation d'astrocytes réactionnels en neurones corticaux fonctionnels et intégrés est effectivement possible en conditions neurodégénératives,

mais également que l'efficacité de reprogrammation augmente avec l'âge des animaux, c'està-dire avec le nombre de glies réactionnelles présentes dans le cortex des animaux mutés [33]. C'est également dans un contexte de gliose réactionnelle massive que nous avons, pour notre part, converti les cellules gliales NG2 en neurones fonctionnels dans le cerveau de souris adultes [39]. Pour induire une réactivité gliale à grande échelle, nous avons réalisé une lésion traumatique sévère par incision chirurgicale dans le cortex cérébral. Nous avons ensuite procédé à la reprogrammation directe des cellules gliales activées en injectant, trois jours plus tard, un rétrovirus encodant les facteurs de transcription Ascll et/ou Sox2. Dans ces conditions, l'expression du seul facteur Sox2 permet la reprogrammation neuronale de cellules gliales activées [39]. L'inefficacité de Sox2 dans le cortex non lésé souligne encore l'importance de l'activation des glies dans l'établissement d'un « état reprogrammable » [39]. L'utilisation du traçage génétique (fate mapping) nous a permis d'identifier les cellules gliales NG2 comme la population cellulaire répondant aux facteurs de transcription. Les neurones ainsi induits sont fonctionnels, capables de générer des potentiels d'action et de s'intégrer dans le réseau neuronal cortical, et ce, en dépit de l'environnement réfractaire que représente la cicatrice gliale [39]. De manière similaire, dans un modèle murin de lésion sévère de la moelle épinière, l'équipe de Chun-Li Zhang rapporte que de nouveaux neurones peuvent être générés à partir d'astrocytes, en réponse à une expression transitoire de Sox2 (sous contrôle du promoteur de la Gfap [glial fibrillary acidic protein]) in vivo [41]. La maturation de ces néo-neurones est lente (par comparaison au développement) mais efficace, et se traduit notamment par l'expression de Synapsin-1, un marqueur de terminaison 
présynaptique, et l'acquisition d'une identité GABAergique ou glutamatergique [41]. Cette maturation est rendue encore plus efficace par un traitement par le VPA [41] (Figure 1B). Si l'identité moléculaire exacte et le profil de projection axonale des neurones ainsi obtenus restent à élucider, ces tout premiers travaux, dans leur ensemble, valident la stratégie de génération de neurones fonctionnels à partir de cellules gliales réactionnelles in vivo, qu'il s'agisse de conditions traumatiques ou neurodégénératives. Les prochaines études devront en outre s'attacher à démontrer les bénéfices fonctionnels cette première approche in situ.

\section{Conversion directe neurone-neurone, premières évidences in vivo}

Si les cellules gliales apparaissent comme les candidats évidents à une conversion directe in situ, qu'en est-il des neurones eux-mêmes ? L'idée peut paraître a priori choquante, puisque ces cellules sont notoirement fragiles, ne se divisent plus et représentent la quintessence même des cellules pleinement différenciées. Sur le plan conceptuel cependant, cibler des neurones sains permettrait de contourner plusieurs problèmes. Si l'on considère le processus de reprogrammation et les changements épigénétiques et transcriptionnels drastiques requis, il apparaît probable que la conversion d'un neurone de type $A$ en neurone de type $B$ serait plus directe, plus rapide, et plus efficace que la conversion d'une cellule d'une autre lignée en neurone. D'autre part, il est clairement établi que les maladies neurodégénératives, pour des raisons encore inconnues, ciblent préférentiellement certains sous-types neuronaux, laissant les populations neuronales voisines, au sein d'une même structure, relativement saines et non affectées. Cibler ces populations neuronales saines permettrait de garantir aux neurones reprogrammés un environnement cellulaire identique à celui de leurs homologues décimés par la maladie, et minimiserait de fait les problèmes de survie, d'intégration et de connectivité, tout en optimisant les chances de ces neurones induits de prendre en charge les fonctions neuronales souhaitées. À notre connaissance, il n'existe aucun exemple de conversion directe in vitro entre deux sous-types neuronaux. En revanche, deux exemples de conversion directe in vivo viennent d'être rapportés.

L'équipe de Denis Jabaudon et nous-mêmes avons testé simultanément la reprogrammation neurone-neurone in vivo [42, 43], en réponse à Fezf2 (Fez family zinc finger 2). Ce facteur de transcription est exprimé par tous les neurones corticofugaux (à projection sous-corticale) des couches $V$ et $V I$ du cortex cérébral, du début de la corticogenèse à l'âge adulte [44], et son expression est nécessaire à la spécification des neurones corticospinaux de la couche $V$ [45-47]. Nous avons précédemment montré que l'expression de Fezf2 suffit à induire la génération ectopique de neurones corticofugaux in vivo, à partir de progéniteurs de neurones cortico-corticaux des couches supérieures du cortex [45] ou de progéniteurs de neurones du striatum et du bulbe olfactif [48]. Les neurones ainsi générés expriment les marqueurs typiques des couches corticales $\mathrm{V}$ et $\mathrm{VI}$, développent une morphologie et une connectivité similaires à leurs homologues corticaux, et adoptent une identité glutamatergique [48]. Ces premiers travaux suggèrent que Fezf2 se comporte comme un « différenciateur terminal », capable à lui seul de conférer à un progéniteur neuronal toutes les caractéristiques qui définissent précisément un sous-type neuronal donné. $\varepsilon n$ utilisant des formes plasmidiques conditionnelles et/ou inductibles de Fezf2, nous avons sélectivement ciblé in vivo les neurones calleux des couches corticales II/III à un stade de neurones postmitotiques embryonnaires, ainsi qu'à un stade de neurones matures postnataux (P3) [43]. Nous montrons que Fezf2 suffit à reprogrammer les neurones calleux postmitotiques en neurones corticofugaux, exprimant en combinaison les marqueurs spécifiques des couches corticales $\mathrm{V}$ et $\mathrm{VI}$, et capables de projeter leurs axones vers des cibles sous-corticales (thalamus) et sous-cérébrales (moelle épinière), typiques des neurones des couches VI et $V$ du cortex, au détriment des connexions corticales (via le corpus calleux) normalement établies par les neurones ciblés [43] (Figure 1C). L'équipe de D. Jabaudon a, quant à elle, choisi de cibler les neurones stellaires de la couche IV du cortex de souriceaux âgés de un jour ( $P 1)$, démontrant que la surexpression de Fezf2 est capable d'induire, dans ces cellules, l'acquisition d'une morphologie, d'un profil électrophysiologique et de connexions émises et reçues typiques des neurones de la couche V [42].

Ces deux études montrent que le neurone, archétype de la cellule différenciée, est beaucoup plus plastique qu'initialement envisagé et peut, à l'instar des cellules gliales, reprogrammer son identité, sa fonction et ses connexions en réponse à des signaux intrinsèques forts. $\varepsilon$ n outre, les résultats obtenus avec Fezf2 suggèrent qu'il est possible d'induire, avec un seul et même facteur de transcription, toutes les caractéristiques qui définissent spécifiquement une sous-population neuronale donnée, à partir d'une cellule d'une autre identité. L'identification de tels « différenciateurs terminaux » pour d'autres sous-types neuronaux d'intérêt thérapeutique permettrait de concevoir des stratégies de reprogrammation garantissant l'obtention d'un soustype neuronal voulu, à partir de cellules (par exemple des glies) rendues capables d'y répondre par une expression transitoire de facteurs neurogéniques plus généraux tels Sox2, Ascll ou encore Neurog2.

\section{Conclusions et perspectives}

Si le tout jeune domaine de la reprogrammation directe dans le SNC semble déjà en pleine effervescence, il lui reste encore à apporter les réponses à un certain nombre de questions fondamentales qui jalonnent les étapes de sa transition du laboratoire de recherche 
au chevet des patients. Quelles cellules cibler? Quels facteurs de transcription utiliser? Quelles seraient les conséquences de la perte des cellules recrutées sur l'homéostasie tissulaire? Quelle méthode employer pour transférer les gènes adéquats au sein des régions d'intérêt chez les patients, et ce, de manière inoffensive? Comment stimuler maturation, survie et activité des néo-neurones dans un microenvironnement non permissif, voire potentiellement nocif ? Comment permettre l'établissement, par les neurones reprogrammés, des connexions appropriées et la restauration des fonctions endommagées?

Il apparaît naturellement nécessaire de poursuivre l'effort mené in vitro, afin de déterminer les meilleures combinaisons de facteurs de transcription permettant d'obtenir, à partir de cellules cérébrales, les sous-types neuronaux les plus significatifs d'un point de vue thérapeutique. II est probable que cela nécessitera la combinaison de facteurs proneurogéniques, capables d'induire une identité neuronale générale, à des « différenciateurs terminaux », capables de conférer les caractéristiques spécifiques d'un sous-type neuronal donné. II nous reste encore beaucoup à apprendre des processus de spécification neuronale au cours du développement pour identifier de tels facteurs.

Les prochaines études in vivo devront, quant à elles, s'atteler à identifier la population cellulaire la plus appropriée pour la reprogrammation. II est probable que la réponse varie en fonction des pathologies. $\varepsilon$ n effet, si la réactivité gliale est immédiate en conditions traumatiques, elle met plus de temps à s'installer en conditions neurodégénératives, et il est possible qu'un nombre conséquent de candidats à la reprogrammation ne soient atteints qu'à des stades trop avancés de la pathologie. Dans de telles conditions, les glies ne seraient peut-être pas les cellules de choix, et les neurones, à l'inverse, pourraient s'avérer d'une grande aide. Enfin, il reste encore à estimer le pourcentage de conversion neuronale optimal, celui qui permettra de restaurer les fonctions perdues sans pour autant créer de nouveaux symptômes neurologiques.

Les efforts de recherche poursuivis dans le domaine de la thérapie génique ont permis d'identifier diverses méthodes d'administration de gènes. Notamment, l'utilisation de virus adéno-associés (AAV), délivrés localement, a permis d'améliorer les symptômes de patients souffrant de cécité congénitale, ou encore de maladie de Parkinson [49]. Parallèlement, le développement de vecteurs non viraux permet à présent d'envisager des méthodes alternatives, potentiellement moins nocives car moins à même de déclencher une réponse inflammatoire. De nombreux vecteurs non viraux font actuellement l'objet d'études cliniques portant sur des pathologies congénitales, cancers et infections virales [50]. Le choix minutieux des méthodes et voies d'administration de facteurs de transcription capables de convertir une population cellulaire donnée en neurones d'intérêt thérapeutique représentera, inévitablement, un élément clé dans le succès de telles approches chez l'homme.

Si le chemin vers l'application thérapeutique de la reprogrammation directe in situ appliquée au SNC semble encore long, des précédents existent déjà dans d'autres organes. Dans l'oreille interne, les cellules de soutien peuvent être converties en cellules ciliées et restaurer l'audition de cochons d'Inde sourds [51]. Les cellules bêta du pancréas peuvent être régénérées chez la souris à partir de cellules exocrines et sécréter de l'insuline, corrigeant ainsi les symptômes du diabète $[52,57]$. Dernièrement, c'est dans le cœur de souris que cette stratégie s'est révélée gagnante, avec la reprogrammation directe de fibroblastes cardiaques en myocytes contractiles, améliorant les fonctions cardiaques suite à un infarctus [53, 54]. L'un des grands défis de l'application de telles approches dans le SNC réside non seulement dans la capacité des neurones reprogrammés à survivre à long terme, mais aussi et surtout à intégrer un réseau neuronal préexistant et à y établir les connexions axonales, souvent sur de très longues distances, nécessaires à la restauration des fonctions nerveuses lésées. Les études menées au début des années 2000 dans le laboratoire de Jeffrey Macklis $[55,56]$, corroborées par nos résultats récents de reprogrammation induite par Fezf2 [43], suggèrent que le cerveau maintient, au-delà du stade du développement, la possibilité d'établir de nouvelles connexions nerveuses, notamment vers la moelle épinière. La régénération du SNC par conversion directe de ses cellules n'appartient plus au registre des utopies, mais apparaît au contraire comme une approche thérapeutique alternative prometteuse et en plein développement. $\diamond$

\section{SUMMARY}

Inducing brain regeneration from within:

in vivo reprogramming of endogenous somatic cells into neurons

In order to overcome the quasi-total inability of the mammalian central nervous system to regenerate in response to injuries, and in parallel to the studies dedicated to prevent neuronal loss under these circumstances, alternative approaches based on the programming of pluripotent cells or the reprogramming of somatic cells into neurons have recently emerged. These uniquely combine growing knowledge of the mechanisms that underlie neurogenesis and neuronal specification during development to the most recent findings of the molecular and epigenetic mechanisms that govern the acquisition and maintenance of cellular identity. Here, we discuss the possibility to instruct the regeneration of the central nervous system from within for therapeutic purposes, in light of the recent works reporting on the generation of neurons by direct conversion of various cerebral cell types in vitro and in vivo. $\diamond$

\section{REMERCIEMENTS}

Nos recherches sont soutenues par l'aide financière de la fondation américaine Citizens United for Research in Epilepsy (CURE), la Fédération pour la recherche sur le cerveau (FRC), la Fondation française pour la recherche sur l'épilepsie (FFRE) pour 
Christophe Heinrich et par l'European research council (ERC), l'Association française contre les myopathies (AFM) et le réseau tri-national des neurosciences du Haut-Rhin (Neurex) pour Caroline Rouaux.

\section{LIENS D'INTÉRÊT}

Les auteurs déclarent n'avoir aucun lien d'intérêt concernant les données publiées dans cet article.

\section{RÉFÉRENCES}

1. Ming GL, Song H. Adult neurogenesis in the mammalian central nervous system. Annu Rev Neurosci $2005 ; 28: 223-50$.

2. Kernie SG, Parent JM. Forebrain neurogenesis after focal Ischemic and traumatic brain injury. Neurobiol Dis $2010 ; 37: 267-74$

3. Wichterle H, Lieberam I, Porter JA, Jessell TM. Directed differentiation of embryonic stem cells into motor neurons. Cell $2002 ; 110: 385-97$

4. Andersson $\varepsilon$, Tryggvason U, Deng $Q$, et al. Identification of intrinsic determinants of midbrain dopamine neurons. Cell $2006 ; 124: 393-405$.

5. Nefzger CM, Haynes JM, Pouton CW. Directed expression of Gata2, Mash1, and Foxa2 synergize to induce the serotonergic neuron phenotype during in vitro differentiation of embryonic stem cells. Stem Cells $2011 ; 29:$ 928-39.

6. Takahashi K, Yamanaka S. Induction of pluripotent stem cells from mouse embryonic and adult fibroblast cultures by defined factors. Cell $2006 ; 126: 663-76$.

7. Davis RL, Weintraub H, Lassar AB. Expression of a single transfected cDNA converts fibroblasts to myo-blasts. Cell 1987 ; $51: 987-1000$.

8. Weintraub H, Tapscott SJ, Davis RL, et al. Activation of muscle-specific genes in pigment, nerve, fat, liver, and fibroblast cell lines by forced expression of MyoD. Proc Natl Acad Sci USA 1989 ; 86 : 5434-38.

9. Choi J, Costa ML, Mermelstein CS, et al. MyoD converts primary dermal fibroblasts, chondroblasts, smooth muscle, and retinal pigmented epithelial cells into striated mononucleated myoblasts and multinucleated myotubes. Proc Natl Acad Sci USA $1990 ; 87$ : 7988-92.

10. Xie H, Ye M, Feng R, Graf T. Stepwise reprogramming of B cells into macrophages. Cell $2004 ; 117$ : 663-76.

11. Feng R, Desbordes SC, Xie H, et al. PU.l and C/EBPalpha/beta convert fibroblasts into macrophage-like cells. Proc Natl Acad Sci USA 2008 ; 105 : 6057-62.

12. Vierbuchen $T$, Ostermeier A, Pang ZP, et al. Direct conversion of fibroblasts to functional neurons by defined factors. Nature $2010 ; 463: 1035-41$.

13. Vierbuchen T, Wernig M. Direct lineage conversions: unnatural but useful? Nat Biotechnol 2011 ; $29: 892-907$.

14. Yang N, Ng YH, Pang ZP, et al. Induced neuronal cells: how to make and define a neuron? Cell Stem Cell $2011 ; 9: 517-27$.

15. Allodi I, Hedlund $\varepsilon$. Directed midbrain and spinal cord neurogenesis from pluripotent stem cells to model development and disease in a dish. Front Neurosci $2014 ; 8: 109$.

16. Anderson S, Vanderhaeghen P. Cortical neurogenesis from pluriotent stem cells : complexity emerging from simplicity. Curr Opin Neurobiol 2014 ; 27C : 151-7.

17. Piang L, Fujita R, Yamashita T, et al. Directed conversion of Alzheimer's disease patient skin fibroblasts into functional neurons. Cell $2011 ; 146: 359-71$.

18. Caiazzo M, Dell'Anno MT, Dvoretskova $\varepsilon$, et al. Direct generation of functional dopaminergic neurons from mouse and human fibroblasts. Nature $2011 ; 476: 224-7$.

19. Son $\varepsilon y$, Ichida JK, Wainger BJ, et al. Conversion of mouse and human fibroblasts into functional spinal motor neurons. Cell Stem Cell $2011 ; 9$ : 205-18.

20. Ang CE, Wernig M. Induced neuronal reprogramming. J Comp Neurol $2014 ; 522$ : 2877-86.

21. Kim J, Su SC, Wang H, et al. Functional integration of dopaminergic neurons directly converted from mouse fibroblasts. Cell Stem Cell $2011 ; 9: 413-9$.

22. Liu X, Li F, Stubblefield $\varepsilon A$, et al. Direct reprogramming of human fibroblasts into dopaminergic neuron-like cells. Cell Res $2012 ; 22: 321-32$.

23. Barker RA, Barrett J, Mason SL, Björkund A. Fetal dopaminergic transplantation trials and the future of neural grafting in Parkinson's disease. Lancet Neurol $2013 ; 12: 84-91$.

24. Sofroniew MV. Molecular dissection of reactive astrogliosis and glial scar formation. Trends Neurosci $2009 ; 32: 638-47$

25. Robel S, Berninger B, Gotz M. The stem cell potential of glia: lessons from reactive gliosis. Nat Rev Neurosci $2011 ; 12: 88-104$

26. Heins N, Malatesta P, Cecconi F, et al. Glial cells generate neurons: the role of the transcription factor Pax6. Nat Neurosci $2002 ; 5: 308-15$

27. Berninger $B$, Costa MR, Koch $U$, et al. Functional properties of neurons derived from in vitro reprogrammed postnatal astroglia. J Neurosci $2007 ; 27: 8654-64$

28. Heinrich C, Blum R, Gascon S, et al. Directing astroglia from the cerebral cortex into subtype specific functional neurons. PLoS Biol $2010 ; 8$ : e1000373.

29. Heinrich C, Gascon S, Masserdotti G, et al. Generation of subtype-specific neurons from postnatal astroglia of the mouse cerebral cortex. Nat Protoc $2011 ; 6: 214-28$
30. Heinrich C, Gotz M, Berninger B. Reprogramming of postnatal astroglia of the mouse neocortex into functional, synapse-forming neurons. Methods $\mathrm{Mol}$ Biol $2012 ; 814: 485-98$

31. Blum R, Heinrich C, Sanchez R, et al. Neuronal network formation from reprogrammed early postnatal rat cortical glial cells. Cereb Cortex 2011 $21: 413-24$.

32. Addis RC, Hsu FC, Wright RL, et al. Efficient conversion of astrocytes to functional midbrain dopaminergic neurons using a single polycistronic vector. PLoS One 2011 ; 6 : e28719.

33. Guo Z, Zhang L, Wu Z, et al. In vivo direct reprogramming of reactive glial cells into functional neurons after brain injury and in an Alzheimer's disease model. Cell Stem Cell 2014 ; 14 : 188-202.

34. Karow M, Sanchez R, Schichor C, et al. Reprogramming of pericyte-derived cells of the adult human brain into induced neuronal cells. Cell Stem Cell $2012 ; 11: 471-6$.

35. Goritz C, Dias D0, Tomilin N, et al. A pericyte origin of spinal cord scar tissue. Science $2011 ; 333: 238-42$

36. Torper 0 , Pfisterer $U$, Wolf DA, et al. Generation of induced neurons via direct conversion in vivo. Proc Natl Acad Sci USA 2013; 110 : 7038-43.

37. Niu $W$, Zang $T$, Zou $Y$, et al. In vivo reprogramming of astrocytes to neuroblasts in the adult brain. Nat Cell Biol 2013; 15 : 1164-75.

38. Graham V, Khudyakov J, Ellis P, Pevny L. SOX2 functions to maintain neural progenitor identity. Neuron $2003 ; 39: 749-65$.

39. Heinrich C, Bergami M, Gascón S, et al. Sox2-mediated conversion of NG2 glia into induced neurons in the injured adult cerebral cortex. Stem Cell Reports $2014 ; 3: 1000-14$

40. Grande A, Sumiyoshi K, Lopez-Juarez A, et al. Environmental impact on direct neuronal reprogramming in vivo in the adult brain. Nat Commun $2013 ; 4: 2373$

41. Su Z, Niu W, Liu ML, et al. In vivo conversion of astrocytes to neurons in the injured adult spinal cord. Nat Commun $2014 ; 5: 3338$

42. De la Rossa A, Bellone C, Golding B, et al. In vivo reprogramming of circuit connectivity in postmitotic neocortical neurons. Nat Neurosci $2013 ; 16$ : 193-200.

43. Rouaux C, Arlotta P. Direct lineage reprogramming of post-mitotic callosal neurons into corticofugal neurons in vivo. Nat Cell Biol 2013; $15: 214-21$.

44. Arlotta P, Molyneaux BJ, Chen J, et al. Neuronal subtype-specific genes that control corticospinal motor neuron development in vivo. Neuron $2005 ; 45$ 207-21.

45. Molyneaux BJ, Arlotta $\mathrm{P}$, Hirata $\mathrm{T}$, et al. Fezl is required for the birth and specification of corticospinal motor neurons. Neuron 2005; $47: 817-31$.

46. Chen JG, Rasin MR, Kwan Ky, Sestan N. Zfp312 is required for subcortical axonal projections and dendritic morphology of deep-layer pyramidal neurons of the cerebral cortex. Proc Natl Acad Sci USA 2005 ; 102 : 17792-7.

47. Chen B, Schaevitz LR, McConnell SK. Fezl regulates the differentiation and axon targeting of layer 5 subcortical projection neurons in cerebral cortex. Proc Natl Acad Sci USA 2013; 102 : 17184-9.

48. Rouaux $C$, Arlotta P. Fezf2 directs the differentiation of corticofugal neurons from striatal progenitors in vivo. Nat Neurosci $2010 ; 13$ : 1345-7.

49. Mingozzi F, High KA. Therapeutic in vivo gene transfer for genetic disease using AAV : progress and challenges. Nat Rev Genet $2011 ; 12$ : 341-55.

50. Yin H, Kanasty RL, Eltoukhy AA, et al. Non-viral vectors for gene-based therapy. Nat Rev Genet $2014 ; 15: 541-55$.

51. Izumikawa M, Minoda R, Kawamoto K, et al. Auditory hair cell replacement and hearing improvement by Atohl gene therapy in deaf mammals. Nat Med $2005 ; 11: 271-6$

52. Zhou $Q$, Brown J, Kanarek $A$, et al. In vivo reprogramming of adult pancreatic exocrine cells to beta-cells. Nature $2008 ; 455: 627-32$.

53. Pian $\mathrm{L}$, Huang $\mathrm{Y}$, Spencer $\mathrm{Cl}$, et al. In vivo reprogramming of murine cardiac fibroblasts into induced cardiomyocytes. Nature 2012 ; 485 : 593-8.

54. Song $\mathrm{K}$, Nam YJ, Luo X, et al. Heart repair by reprogramming non-myocytes with cardiac transcription factors. Nature 2012 ; 485 : 599-604.

55. Magavi SS, Leavitt BR, Macklis JD. Induction of neurogenesis in the cortex of adult mice. Nature $2000 ; 405: 951-5$.

56. Chen J, Magavi SS, Macklis JD. Neurogenesis of corticoscpinal motor neurons extending spinal projections in adult mice. Proc Natl Acad Sci USA 2004 $101: 16357-62$

57. Vieira A, Druelle N, Courtney M, et al. Reprogrammation des cellules pancréatiques en cellules $\beta$. Med Sci (Paris) $2013 ; 29: 749-55$

\section{TIRÉS À PART}

C. Rouaux et C. Heinrich 